A Guide for Students and Researchers

\title{
Statistical Mechanics
}

Simple 
This page is intentionally left blank 
A Guide for Students and Researchers Statistical Mechanics Simple

Daniel C. Mattis

University of Utah, USA 


\title{
Published by
}

World Scientific Publishing Co. Pte. Ltd.

5 Toh Tuck Link, Singapore 596224

USA office: Suite 202, 1060 Main Street, River Edge, NJ 07661

UK office: 57 Shelton Street, Covent Garden, London WC2H 9HE

\author{
Library of Congress Cataloging-in-Publication Data \\ Mattis, Daniel Charles, 1932- \\ p. $\mathrm{cm}$. \\ Includes bibliographical references and index. \\ ISBN 981-238-165-1 -- ISBN 981-238-166-X (pbk.) \\ 1. Statistical mechanics. I. Title. \\ QC174.8.M365 2003 \\ $530.13--\mathrm{dc} 21$ \\ 2003042254

\section{British Library Cataloguing-in-Publication Data} \\ A catalogue record for this book is available from the British Library.
}

Statistical mechanics made simple : a guide for students and researchers / Daniel C. Mattis.

Copyright $\odot 2003$ by World Scientific Publishing Co. Pte. Ltd.

All rights reserved. This book, or parts thereof, may not be reproduced in any form or by any means, electronic or mechanical, including photocopying, recording or any information storage and retrieval system now known or to be invented, without written permission from the Publisher.

For photocopying of material in this volume, please pay a copying fee through the Copyright Clearance Center, Inc., 222 Rosewood Drive, Danvers, MA 01923, USA. In this case permission to photocopy is not required from the publisher. 


\section{Contents}

Preface ix

Introduction: Theories of Thermodynamics, Kinetic Theory and

Statistical Mechanics

Chapter 1 Elementary Concepts in Statistics and Probability 1

1.1. The Binomial Distribution . . . . . . . . . . . . . . . 1

1.2. Length of a Winning Streak . . . . . . . . . . . . 3

1.3. Brownian Motion and the Random Walk . . . . . . . . . . . 4

1.4. Poisson versus Normal (Gaussian) Distributions . . . . . . . . 5

1.5. Central Limit Theorem (CLT) . . . . . . . . . . . . . . 9

1.6. Multinomial Distributions, Statistical Thermodynamics . . . . . 12

1.7. The Barometer Equation . . . . . . . . . . . . . . . 14

1.8. Other Distributions . . . . . . . . . . . . . . . . . . . 14

Chapter 2 The Ising Model and the Lattice Gas 17

2.1. Some Background and Motivation . . . . . . . . . . . . 17

2.2. First-Principles Statistical Theory of Paramagnetism . . . . . . 18

2.3. More on Entropy and Energy . . . . . . . . . . . . . . . . . 21

2.4. Some Other Relevant Thermodynamic Functions . . . . . . . . 21

2.5. Mean-Field Theory, Stable and Metastable Solutions . . . . . 23

2.6. The Lattice Gas . . . . . . . . . . . . . . . . . . . . 26

2.7. The Nearest-Neighbor Chain: Thermodynamics in 1D . . . 26

2.8. The Disordered Ising Chain . . . . . . . . . . . 28

2.9. Other Magnetic Systems in One Dimension . . . . . . . . 28 
$\begin{array}{lll}\text { Chapter } 3 & \text { Elements of Thermodynamics } & 31\end{array}$

3.1. The Scope of Thermodynamics . . . . . . . . . . . . . 31

3.2. Equations of State and Some Definitions . . . . . . . . . . 32

3.3. Maxwell Relations . . . . . . . . . . . . . . . . . 35

3.4. Three Important Laws of Thermodynamics . . . . . . . . . . 35

3.5. The Second Derivatives of the Free Energy . . . . . . . . . . . 38

3.6. Phase Diagrams for the van der Waals Gas . . . . . . . . . . 39

3.7. Clausius-Clapeyron Equation . . . . . . . . . . . 43

3.8. Phase Transitions . . . . . . . . . . . . . . . . 46

3.9. The Carnot Cycle . . . . . . . . . . . . . . . . . . . . 49

3.10. Superconductivity . . . . . . . . . . . . . 51

Chapter 4 Statistical Mechanics 55

4.1. The Formalism - and a False Start . . . . . . . . . . 55

4.2. Gibbs' Paradox and Its Remedy . . . . . . . . . . . . . 58

4.3. The Gibbs Factor . . . . . . . . . . . . . . . . . . . 59

4.4. The Grand Ensemble . . . . . . . . . . . . . . . . . . . 60

4.5. Non-Ideal Gas and the 2-Body Correlation Function . . . . . 62

4.6. The Virial Equation of State . . . . . . . . . . . . . . . . 64

4.7. Weakly Non-Ideal Gas . . . . . . . . . . . . . . . 65

4.8. Two-body Correlations . . . . . . . . . . . . . . 68

4.9. Configurational Partition Function in 1D . . . . . . . . 73

4.10. One Dimension versus Two . . . . . . . . . . . 75

4.11. Two Dimensions versus Three: The Debye-Waller Factors . . . 77

Chapter 5 The World of Bosons $\quad 83$

5.1. Two Types of Bosons and Their Operators . . . . . . . . . . 83

5.2. Number Representation and the Many-Body Problem . . . . 86

5.3. The Adiabatic Process and Conservation of Entropy . . . . . . 88

5.4. Many-Body Perturbations . . . . . . . . . . . . . . . . 89

5.5. Photons . . . . . . . . . . . . . . . . . 90

$5.6 . \quad$ Phonons . . . . . . . . . . . . . . . . 93

5.7. Ferromagnons . . . . . . . . . . . . . . 96

5.8. Conserved Bosons and the Ideal Bose Gas . . . . . . . . . . . . 99

5.9. Nature of "Ideal" Bose-Einstein Condensation . . . . . . . . . . 101

5.10. Ideal Bose-Einstein Condensation in Low Dimensions . . . . . . 104

5.11. Consequences of a Hard Core Repulsion in 1D . . . . . . . . 106

5.12. Bosons in 3D Subject to Weak Two-Body Forces . . . . . . . . 109

5.13. Superfluid Helium (He II) . . . . . . . . . . . . . . . . 114 
Chapter 6 All About Fermions: Theories of Metals, Superconductors, Semiconductors

6.1. Fermi-Dirac Particles . . . . . . . . . . . . . . . . . . . . 119

6.2. Slater Determinant: The Ground State . . . . . . . . . . 120

6.3. Ideal Fermi-Dirac Gas . . . . . . . . . . . . . . . . . . . . . 121

6.4. Ideal Fermi-Dirac Gas with Spin . . . . . . . . . . . . 123

6.5. Fermi Integrals . . . . . . . . . . . . . . . . . . . . 124

6.6. Thermodynamic Functions of an Ideal Metal . . . . . . . . . . 125

6.7. Quasiparticles and Elementary Excitations . . . . . . . . . . 128

6.8. Semiconductor Physics: Electrons and Holes . . . . . . . . . . 130

6.9. n-Type Semiconductor Physics: The Statistics . . . . . . . . . 131

6.10. Correlations and the Coulomb Repulsion . . . . . . . . . . . 132

6.11. Miscellaneous Properties of Semiconductors . . . . . . . . . 135

6.12. Aspects of Superconductivity: Cooper Pairs . . . . . . . . . 137

6.13. Aspects of BCS Theory . . . . . . . . . . . . . . . . . 140

6.14. Contemporary Developments in Superconductivity . . . . . 146

$\begin{array}{lll}\text { Chapter } 7 & \text { Kinetic Theory } & 149\end{array}$

7.1. Scope of This Chapter . . . . . . . . . . . . . . . . . . 149

7.2. Quasi-Equilibrium Flows and the Second Law . . . . . . . . . 150

7.3. The Collision Integral . . . . . . . . . . . . . . . 151

7.4. Approach to Equilibrium of a "Classical" Non-Ideal Gas . . . 154

7.5. A New Look at "Quantum Statistics" . . . . . . . . . . . . . 156

7.6. Master Equation: Application to Radioactive Decay . . . . . . . 158

7.7. Boltzmann Equation . . . . . . . . . . . . . . . 160

7.8. Electrical Currents in a Low-Density Electron Gas . . . . . 162

7.9. Diffusion and the Einstein Relation . . . . . . . . . . 165

7.10. Electrical Conductivity of Metals . . . . . . . . . . 165

7.11. Exactly Solved "Backscattering" Model . . . . . . . . . . . . 166

7.12. Electron-Phonon Scattering . . . . . . . . . . . . . . . 169

7.13. Approximating the Boltzmann Equation . . . . . . . . 170

7.14. Crossed Electric and Magnetic Fields . . . . . . . . . . . 171

7.15. Propagation of Sound Waves in Fluids . . . . . . . . . 173

7.16. The Calculations and Their Result . . . . . . . . . . 178

Chapter 8 The Transfer Matrix 183

8.1. The Transfer Matrix and the Thermal Zipper . . . . . . . . 183

8.2. Opening and Closing a "Zipper Ladder" or Polymer . . . . . 186

8.3. The Full Zipper $(N>2) \ldots \ldots \ldots \ldots \ldots$ 
8.4. The Transfer Matrix and Gaussian Potentials . . . . . . . . 191

8.5. Transfer Matrix in the Ising Model . . . . . . . . . . . . . 192

8.6. The Ising Ladder or Polymer . . . . . . . . . . . . . . . 195

8.7. Ising Model on the Isotropic Square Lattice (2D) $\ldots \ldots \ldots$

8.8. The Phase Transition . . . . . . . . . . . . . . . . . . . . 201

8.9. A Question of Long-Range Order . . . . . . . . . . . . . . 202

8.10. Ising Model in 2D and 3D . . . . . . . . . . . . . 204

8.11. Antiferromagnetism and Frustration . . . . . . . . 206

8.12. Maximal Frustration . . . . . . . . . . . . . . . . . 208

8.13. Separable Model Spin-Glass without Frustration . . . . . . . 210

8.14. Critical Phenomena and Critical Exponents . . . . . . . . 212

8.15. Potts Models . . . . . . . . . . . . . . . . 215

\section{Chapter 9 Some Uses of Quantum Field Theory in} Statistical Physics

9.1. Outline of the Chapter . . . . . . . . . . . . . . . . . . 219

9.2. Diffusion on a Lattice: Standard Formulation . . . . . . . . . 219

9.3. Diffusion as Expressed in QFT . . . . . . . . . . . . 222

9.4. Diffusion plus One-Body Recombination Processes . . . . . . 225

9.5. Diffusion and Two-Body Recombination Processes . . . . . 226

9.6. Questions Concerning Long-Range Order . . . . . . . . . . . . . 228

9.7. Mermin-Wagner Theorem . . . . . . . . . . . . . . 230

9.8. Proof of Bogolubov Inequality . . . . . . . . . . . . . . 233

9.9. Correlation Functions and the Free Energy . . . . . . . . . . 234

9.10. Introduction to Thermodynamic Green's Functions . . . . . 237

Index 


\section{Preface}

I dedicate this book to those generations of students who suffered through endless revisions of my class notes in statistical mechanics and, through their class participation, homework and projects, helped shape the material.

My own undergraduate experience in thermodynamics and statistical mechanics, a half-century ago at MIT, consisted of a single semester of Sears' Thermodynamics (skillfully taught by the man himself.) But it was a subject that seemed as distant from "real" physics as did poetry or French literature. Graduate study at the University of Illinois in Urbana-Champaign was not that different, except that the course in statistical mechanics was taught by the brilliant lecturer Francis Low the year before he departed for... MIT. I asked my classmate J.R. Schrieffer, who presciently had enrolled in that class, whether I should chance it later with a different instructor. He said not to bother - that he could explain all I needed to know about this topic over lunch.

On a paper napkin, Bob wrote " $e^{-\beta H " . ~ " T h a t ' s ~ i t ~ i n ~ a ~ n u t s h e l l ! " ~ " S u r e l y ~}$ you must be kidding, Mr Schrieffer," I replied (or words to that effect.) "How could you get the Fermi-Dirac distribution out of THAT? "Easy as pie," was the reply ${ }^{\mathrm{a}}$... and I was hooked.

I never did take the course, but in those long gone days it was still possible to earn a Ph.D. without much of a formal education. Schrieffer, of course, with John Bardeen and Leon Cooper, went on to solve the statistical mechanics of superconductors and thereby earn the Nobel prize.

The standard book on statistical physics in the late 1950's was by T. L. Hill. It was recondite but formal and dry. In speaking of a different text that was feebly attempting the same topic, a wit quipped that "it was not worth

a See Chapter 6. 
a bean of Hill's." Today there are dozens of texts on the subject. Why add one more?

In the early 1960's, while researching the theory of magnetism, I determined to understand the two-dimensional Ising model that had been so brilliantly resolved by Lars Onsager, to the total and utter incomprehension of just about everyone else. Ultimately, with the help of Elliot Lieb and Ted Schultz (then my colleagues at IBM's research laboratory,) I managed to do so and we published a reasonably intelligible explanation in Reviews of Modern Physics. This longish work - parts of which appar in Chapter 8 - received an honorable mention almost 20 years later, in the 1982 Nobel lecture by Kenneth G. Wilson, who wrote:

"In the summer of 1966 I spent a long time at Aspen. While there I carried out a promise I had made to myself while a graduate student, namely [to work] through Onsager's solution of the two- dimensional Ising model. I read it in translation, studying the field-theoretic form given in Lieb, Mattis and Schultz ['s paper.] When I entered graduate school I had carried out the instructions given to me by my father and had knocked on both Murray Gell-Mann's and [Richard] Feynman's doors and asked them what they were currently doing. Murray wrote down the partition function of the threedimensional Ising model and said it would be nice if I could solve it.... Feynman's answer was "nothing." Later, Jon Mathews explained some of Feynman's tricks for reproducing the solution for the two-dimensional Ising model. I didn't follow what Jon was saying, but that was when I made my promise.... As I worked through the paper of Mattis, Lieb and Schultz I realized there should be applications of my renormalization group ideas to critical phenomena..."b

Recently, G. Emch has reminded me that at the very moment Wilson was studying our version of the two-dimensional Ising model I was attending a large IUPAP meeting in Copenhagen on the foundations and applications of statistical mechanics. My talk had been advertised as, "The exact solution of the Ising model in three dimensions" and, needless to say, it was well attended. I did preface it by admitting there was no exact solution but that - had the airplane taking me to Denmark crashed - the title alone would have earned me a legacy worthy of Fermat. Although it was anticlimactic, the actual talk ${ }^{\mathrm{c}}$ demonstrated that in 5 spatial dimensions or higher, mean-field theory prevails.

\footnotetext{
${ }^{\mathrm{b}}$ From Nobel Lectures in Physics (1981-1990), published by World Scientific.

' It appeared in the Proceedings with a more modest title befitting a respectable albeit approximate analysis.
} 
In the present book I have set down numerous other topics and techniques, much received wisdom and a few original ideas to add to the "hill of beans." Whether old or new, all of it can be turned to advantage. My greatest satisfaction will be that you read it here first.

D.C.M.

Salt Lake City, 2003 
This page is intentionally left blank 


\section{Introduction: Theories of Thermodynamics, Kinetic Theory and Statistical Mechanics}

Despite the lack of a reliable atomic theory of matter, the science of Thermodynamics flourished in the 19th Century. Among the famous thinkers it attracted, one notes William Thomson (Lord Kelvin) after whom the temperature scale is named, and James Clerk Maxwell. The latter's many contributions include the "distribution function" and some very useful differential "relations" among thermodynamic quantities (as distinguished from his even more famous "equations" in electrodynamics). The Maxwell relations set the stage for our present view of thermodynamics as a science based on function theory while grounded in experimental observations.

The kinetic theory of gases came to be the next conceptual step. Among pioneers in this discipline one counts several unrecognized geniuses, such as J. J. Waterston who - thanks to Lord Rayleigh - received posthumous honors from the very same Royal Society that had steadfastly refused to publish his works during his lifetime. Ludwig Boltzmann committed suicide on September 5, 1906, depressed - it is said - by the utter rejection of his atomistic theory by such colleagues as Mach and Ostwald. Paul Ehrenfest, another great innovator, died by his own hand in 1933. Among 20th Century scientists in this field, a sizable number have met equally untimely ends. So "now", (here we quote from a well-known and popular text ${ }^{\mathrm{a}}$ ) "it is our turn to study statistical mechanics".

The postulational science of Statistical Mechanics - originally introduced to justify and extend the conclusions of thermodynamics but nowadays extensively studied and used on its own merits - is entirely a product of the 20th Century. Its founding fathers include Albert Einstein (who, among his many other contributions, made sense out of Planck's Law) and J. W. Gibbs, whose formulations of phase space and entropy basically

${ }^{a}$ D. H. Goodstein, States of Matter, Dover, New York, 1985. 
anticipated quantum mechanics. Many of the pioneers of quantum theory also contributed to statistical mechanics. We recognize this implicitly whenever whenever we specify particles that satisfy "Fermi-Dirac" or "BoseEinstein" statistics, or when we solve the "Bloch equation" for the density matrix, or when evaluating a partition function using a "Feynman path integral".

In its most simplistic reduction, thermodynamics is the study of mathematical identities involving partial derivatives of well defined functions. These relate various macroscopic properties of matter: pressure, temperature, density, magnetization, etc., to one another. Phase transitions mark the discontinuities of one or more of these functions and serve to separate distinct regions (e.g. vapor from solid) in the variables' phase space. Kinetic theory seeks to integrate the equations of motion of a many-body system starting from random initial conditions, thereby to construct the system's thermodynamic properties. Finally, statistical mechanics provides an axiomatic foundation for the preceding while allowing a wide choice of convenient calculational schemes.

There is no net flow of matter nor of charged particles in thermodynamic equilibrium. Away from equilibrium but in or near steady state, the Boltzmann equation (and its quantum generalizations by Kubo and others) seeks to combine kinetic theory with statistical mechanics. This becomes necessary in order to explain and predict transport phenomena in a non-ideal medium, or to understand the evolution to equilibrium when starting from some arbitrary initial conditions. It is one of the topics covered in the present text.

Any meaningful approach revolves about taking $N$, the number of distinct particles under consideration, to the limit $N \rightarrow \infty$. This is not such a dim idea in light of the fact that Avogadro's number, $N_{A}=6.022045 \times 10^{23} \mathrm{per}$ mole. ${ }^{b}$

Taking advantage of the simplifications brought about by the law of large numbers and of some 18th Century mathematics one derives the underpinnings for a science of statistical mechanics and, ultimately, finds a theoretical justification for some of the dogmas of thermodynamics. In the 9 chapters to follow we see that a number of approximate relations at small values of $N$ become exact in the "thermodynamic limit" (as the procedure of taking the

\footnotetext{
${ }^{\mathrm{b}} \mathrm{A}$ mole is the amount of a substance that contains as many elementary entities as there are carbon atoms in $12 \mathrm{gm}$ of Carbon 12. E.g.: 1 mole of electrons $\left(\mathrm{e}^{-}\right)$consists of $N_{A}$ particles of total mass $5.4860 \times 10^{-4} \mathrm{gm}$ and total charge $-96.49 \times 10^{3}$ coulombs.
} 
limit $N \rightarrow \infty$ is now known in all branches of physics, including many-body physics and quantum field theory).

Additionally we shall study the fluctuations $O(\sqrt{N})$ in macroscopic $O(N)$ extensive quantities, for "one person's noise is another person's signal". Even when fluctuations are small, what matters most is their relation to other thermodynamic functions. For example, the "noise" in the internal energy, $\left\langle E^{2}\right\rangle-\langle E\rangle^{2}$, is related to the same system's heat capacity $d E / d T$. Additional examples come under the rubric of the "fluctuation-dissipation" theorem.

With Bose-Einstein condensation, "high"-temperature superconductivity, "nanophysics", "quantum dots", and "colossal" magnetoresistance being the order of the day, there is no lack of contemporary applications for the methods of statistical physics. However, first things first. We start the exposition by laying out and motivating the fundamentals and methodologies that have "worked" in such classic systems as magnetism and the non-ideal gas. Once mastered, these reductions should allow one to pose more contemporary questions. With the aid of newest techniques - some of which are borrowed from quantum theory - one can supply some of the answers and, where the answers are still lacking, the tools with which to obtain them. The transition from "simple" statistical mechanics to the more sophisticated versions is undertaken gradually, starting from Chapter 4 to the concluding chapters of the book. The requisite mathematical tools are supplied as needed within each self-contained chapter.

The book was based on the needs of physics graduate students but it is designed to be accessible to engineers, chemists and mathematicians with minimal backgrounds in physics. Too often physics is taught as an idealized science, devoid of statistical uncertainties. An elementary course in thermodynamics and statistical physics can remedy this; Chapters $1-4$ are especially suitable for undergraduates aspiring to be theoreticians. Much of the material covered in this book is suitable for self-study but all of it can be used as a classroom text in a one-semester course.

Based in part on lecture notes that the author developed during a decade of teaching this material, the present volume seeks to cover many essential physical concepts and theoretical "tricks" as they have evolved over the past two centuries. Some theories are just mentioned while others are developed in great depth, the sole criterion being the author's somewhat arbitrary opinion of the intellectual depth of the posed problem and of the elegance of its resolution. Here, function follows form.

Specifically, Chapters 1 and 2 develop the rudiments of a statistical science, touching upon metastable states, phase transitions, critical 
exponents and the like. Applications to magnetism and superconductivity are included $a b$ initio. Chapter 3 recapitulates thermodynamics in a form that invites comparison with the postulational statistical mechanics of Chapter 4. van der Waals gas is studied and then compared to the exactly solved Tonks' gas. Chapters 5 and 6 deal, respectively, with the quantum statistics of bosons and fermions and their various applications. We distinguish the two principal types of bosons: conserved or not. The notion of "quasiparticles" in fermion systems is stressed. We touch upon semiconductor physics and the rôle of the chemical potential $\mu(T)$ in $n$-type semiconductors, analyzing the case when ionized donors are incapable of binding more than one excess electron due to 2-body forces. Chapter 7 presents the kinetic theory of dilute gases. Boltzmann's $H$-function is used to compute the approach to thermodynamic equilibrium and his eponymous equation is transformed into an eigenvalue problem in order to solve for the dispersion and decay of sound waves in gases.

Chapter 8 develops the concept of the transfer matrix, including an Onsager-type solution to the two-dimensional Ising model. Exact formulas are used to calculate the critical exponents of selected second-order phase transitions. The concept of "frustration" is introduced and the transfer matrix of the "fully frustrated" two-dimensional Ising model is diagonalized explicitly. A simplified model of fracture, the "zipper", is introduced and partly solved; in the process of studying this "classical" system, we learn something new about the equations of continuity in quantum mechanics!

Chapter 9, the last, is devoted to more advanced techniques: Doi's field-theoretic approach to diffusion-limited chemical reactions is one and the Green's functions theory of the many-body problem is another. As illustrations we work out the eigenvalue spectrum of several special models including that of a perfectly random Hamiltonian.

Additional models and calculations have been relegated to the numerous problems scattered throughout the text, where you, the reader, can test your mastery of the material. But despite coverage of a wealth of topics this book remains incomplete, as any text of normal length and scope must be. It should be supplemented by the monographs and review articles on critical phenomena, series expansions, reaction rates, exact methods, granular materials, etc., found on the shelves of even the most modest physics libraries. If used to good advantage, the present book could be a gateway to these storehouses of knowledge and research. 